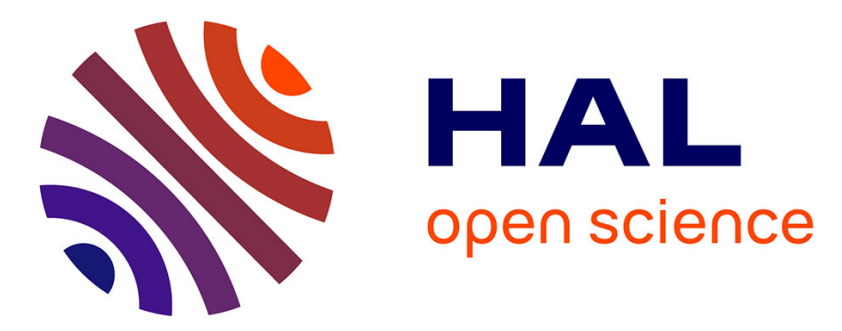

\title{
Coefficient determination via asymptotic spreading speeds
}

Michel Cristofol, Isma Kaddouri, Grégoire Nadin, Lionel Roques

\section{To cite this version:}

Michel Cristofol, Isma Kaddouri, Grégoire Nadin, Lionel Roques. Coefficient determination via asymptotic spreading speeds. Inverse Problems, 2014, 30 (3), pp.035005. 10.1088/0266-5611/30/3/035005 . hal-01072248

\section{HAL Id: hal-01072248 \\ https://hal.science/hal-01072248}

Submitted on 7 Oct 2014

HAL is a multi-disciplinary open access archive for the deposit and dissemination of scientific research documents, whether they are published or not. The documents may come from teaching and research institutions in France or abroad, or from public or private research centers.
L'archive ouverte pluridisciplinaire HAL, est destinée au dépôt et à la diffusion de documents scientifiques de niveau recherche, publiés ou non, émanant des établissements d'enseignement et de recherche français ou étrangers, des laboratoires publics ou privés.

\section{(1) (1) $\$$}

Distributed under a Creative Commons Attribution - NonCommercial - NoDerivatives 44.0 


\title{
Coefficient determination via asymptotic spreading speeds
}

\author{
Michel Cristofol ${ }^{1}$, Isma Kaddouri ${ }^{1,2}$, Grégoire Nadin ${ }^{3}$ and \\ Lionel Roques ${ }^{4}$ \\ 1 Aix-Marseille University, LATP, UMR 7353, France \\ 2 University of Sciences and Technologie Houari Boumediene, Faculty of \\ mathematics, AMNEDP, Algeria \\ 3 University Paris 6, CNRS, laboratoire Jacques-Louis Lions, France \\ 4 UR 546 Biostatistique et Processus Spatiaux, INRA, F-84000 Avignon, France \\ E-mail: ${ }^{1}$ michel.cristofol@univ-amu.fr, ${ }^{2}$ isma.kaddouri@gmail.com, \\ ${ }^{3}$ nadin@ann.jussieu.fr, ${ }^{4}$ lionel.roques@avignon.inra.fr
}

\begin{abstract}
In this paper, we analyze the inverse problem of determining the reaction term $f(x, u)$ in reaction-diffusion equations of the form $\partial_{t} u-D \partial_{x x} u=f(x, u)$, where $f$ is assumed to be periodic with respect to $x \in \mathbb{R}$. Starting from a family of exponentially decaying initial conditions $u_{0, \lambda}$, we show that the solutions $u_{\lambda}$ of this equation propagate with constant asymptotic spreading speeds $w_{\lambda}$. Our main result shows that the linearization of $f$ around the steady state $0, \partial_{u} f(x, 0)$, is uniquely determined (up to a symmetry) among a subset of piecewise linear functions, by the observation of the asymptotic spreading speeds $w_{\lambda}$.
\end{abstract}

\section{Introduction}

This paper is devoted to the reconstruction of the linear part $\partial_{u} f(x, 0)$ of the reaction term $f(x, u)$ in the following reaction-diffusion problem:

$$
\text { (P) }\left\{\begin{array}{l}
\partial_{t} u-D \partial_{x x} u=f(x, u), t>0, x \in \mathbb{R} \\
u(0, x)=u_{0}(x) \geq 0, x \in \mathbb{R}
\end{array}\right.
$$

This equation describes the space-time evolution of a concentration $u(t, x)$ in a heterogeneous excitable environment $[4,47]$. It appears in several fields of applications such as physics, in combustion flame propagation models [9], in chemistry [11], in ecology, to study the dynamics of a population $[36,44]$ as well as in population genetics $[2,3,40]$.

For Kolmogorov-Petrovsky-Piskunov type (KPP) nonlinearities (see definition below), it is well known that the solution $u(t, x)$ of the problem $(P)$ mainly depends on the reaction term $f(x, u)$ through its linearization $\partial_{u} f(x, 0)$ around the steady state 0 (see for exemple $[7,13])$. In population dynamics, this term represents the intrinsic 
growth rate of a population. The persistence or the extinction of the population and its rate of range expansion only depend on $f(x, u)$ through this term $\partial_{u} f(x, 0)$ $[4,7,8,22,27]$. In practice, this term is often unknown or partially known.

In this work, we assume that $D>0$, and that $x \mapsto f(x, u)$ is a 1 -periodic reaction term, that is:

$$
\forall x \in \mathbb{R}, \forall s \in \mathbb{R}, f(x, s)=f(x+1, s) .
$$

The reconstruction of coefficients for parabolic equations is a widely studied problem. Several works are done in the case of parabolic operators defined on an open bounded set (see among others $[1,15,20,21,30,35,37]$ ). However in the unbounded case considered here, there are few references $([31,33])$ about coefficient identification.

In the case of bounded domains, several kinds of observations can be used for the determination of a coefficient. Many results are based on a method involving Carleman inequalities, which has been introduced in the seminal paper of Bukhgeim and Klibanov [12]. This method involves, in addition to localized observations of the solution $u(t, x)$ of the considered problem, an observation of the solution on the whole domain of study, at some fixed time. Following the approach by Imanuvilov and Yamamoto [30] for the reconstruction of a potential in the linear case, several results have been obtained in the nonlinear case $[17,18,19,43]$. More recently, other methods based on the maximum principle and the Hopf's lemma allowed to obtain uniqueness results using only pointwise observations in the one-dimensional case [16, 38, 39].

The study of the direct problem $(P)$ has been widely developed recently (see e.g. $[4,5,6,7,10,45,46])$. The paper [5] is devoted to some nonlinear propagation phenomena in periodic and more general domains, for reaction-diffusion equations with KPP nonlinearities [34]. In [10], the authors establish spreading properties for the solutions of equations of the form $\partial_{t} u-a(x) \partial_{x x} u-q(x) \partial_{x} u=f(x, u)$. A common point of these works concerns the precise study of the speed of propagation of the solutions and of the asymptotic spreading speed.

On the other hand, the inverse problems associated with this class of equations have been little studied. In [14], the author treated the case of a linear parabolic operator in $\mathbb{R}^{n}$, associated with boundary and initial conditions which lead to a periodic solution. Then the problem stated in $\mathbb{R}^{n}$ can be easily written into a problem stated in a bounded cell. The observations required to solve this inverse problem have to be measured on a set which includes all the edges of the cell which induces a strong constraint. In a recent work [31] the authors have improved this result by considering a nonlinear inverse parabolic problem with non-smooth coefficients and have obtained a more general reconstruction result without the above-mentioned constraint on the observation set.

Here, the initial condition $u_{0}$ is not necessarily periodic, and the solution $u(t, x)$ is therefore non-periodic in general. In this context, we use a new type of observations, which are based on the spreading properties of the solutions of the Cauchy problem $(P)$. Before going further on, we recall the classical Kolmogorov-Petrovsky-Piskunov 
assumptions (denoted by KPP in reference to the seminal work [34]):

$$
\left\{\begin{array}{l}
\forall x \in \mathbb{R}, f(x, 0)=0, \\
\exists M>0, \text { such that for } s \geq M, \text { and } \forall x \in \mathbb{R}, f(x, s) \leq 0, \\
\forall x \in \mathbb{R}, s \mapsto \frac{f(x, s)}{s} \text { is decreasing for } s>0 .
\end{array}\right.
$$

Under these hypotheses, the 1-periodicity assumption (1), and assuming that the steady state 0 is linearly unstable (see Proposition 2.1), it is known that the solution of the Cauchy problem $(P)$, starting with a compactly supported or Heaviside initial condition, propagates (to the right) with a finite asymptotic spreading speed $w^{*}[22]$ in the following sense:

$$
\left\{\begin{array}{l}
\lim _{t \rightarrow+\infty} u(t, x+c t)=0, \forall c>w^{*}, x \in \mathbb{R}, \\
\liminf _{t \rightarrow+\infty} u(t, x+c t)>0, \forall 0 \leq c<w^{*}, x \in \mathbb{R} .
\end{array}\right.
$$

This means that an observer moving to the right with a speed $c$ larger than $w^{*}$ will see the solution converge to 0 , whereas if he moves with a speed smaller than $w^{*}$, he will see the solution remain above some positive constant. The existence of this constant asymptotic spreading speed, as well as the existence of traveling wave solutions which propagate with constant speeds $c \geq w^{*}[4,8,45]$, are the key features which contributed to the success of the reaction-diffusion framework in applied sciences $[36,42,44]$ since the pioneering work [34]. In particular, whenever the parameters $D, \partial_{u} f(x, 0)$ and $u_{0}$ in $(P)$ are properly fitted to experimental data, the predicted asymptotic spreading speed $w^{*}$ is often in accordance with observations of species range expansions [42]. It is therefore natural to consider the inverse problem of coefficient determination in equation $(P)$, based on observations of the spreading properties of its solution $u(t, x)$.

The main objective of this paper is to determine the linear part

$$
r(x)=\partial_{u} f(x, 0)
$$

of the reaction term $f(x, u)$, using observations of the spreading speed of the solution of the Cauchy problem $(P)$. In that respect, instead of considering compactly supported

or Heaviside initial conditions, we consider a 1-parameter family of initial conditions $\left(u_{0, \lambda}\right)_{\lambda \in I \subset(0,+\infty)}$, which decay like $e^{-\lambda x}$ as $x \rightarrow+\infty$, and we use the associated asymptotic spreading speeds $w_{\lambda}$ as observations.

The outline of the paper is as follows. In Section 2, we give our assumptions and we state our main result, in Sections 3-5 we detail the proofs of our results.

\section{Assumptions and main results}

\subsection{Assumptions on $f$}

In addition to the periodicity assumption (1) and the KPP assumption (2), we assume that the nonlinearity $(x, u) \mapsto f(x, u)$ is of class $\mathcal{C}^{0, \alpha}$ with respect to $x$ locally uniformly 
in $u \in \mathbb{R}$, and of class $\mathcal{C}^{1}$ with respect to $u$. Besides, we assume that the linear part of the reaction term, $r(x)=\partial_{u} f(x, 0)$ belongs to the function space $\mathcal{M}_{1}$ defined by:

$$
\mathcal{M}_{1}=\left\{1 \text {-periodic function } \rho \in \mathcal{C}^{0}(\mathbb{R}) \text {, linear in }[0, \theta) \text { and }[\theta, 1)\right\},
$$

for some (known) constant $\theta \in(0,1)$. In other words, $r(x)$ is a piecewise linear function.

\subsection{Stationary states: existence, uniqueness and stability}

Under our assumptions on the reaction term $f$ and for continous and bounded initial conditions $u_{0}$, it is known that the solution of the Cauchy problem $(P)$ converges when $t \rightarrow+\infty$ to a nonnegative stationary solution, that is, a solution $p \geq 0$ of the problem:

$$
-D \partial_{x x} p=f(x, p), x \in \mathbb{R} .
$$

More precisely, in [7] the authors have given a necessary and sufficient condition for the existence of a positive stationary state $p$. This condition is based on the description of the linear stability of the steady state 0 , i.e., on the sign of the principal eigenvalue of the linear operator $L_{0}$ :

$$
L_{0}: \psi \mapsto-D \partial_{x x} \psi-r(x) \psi,
$$

with periodicity assumptions on $\psi$. We recall that, from the Krein-Rutman Theorem [24], there exists a unique $k_{0} \in \mathbb{R}$ and a unique function $\psi_{0} \in \mathcal{C}^{2}(\mathbb{R})$ such that

$$
\left\{\begin{array}{l}
-D \partial_{x x} \psi_{0}-r(x) \psi_{0}=k_{0} \psi_{0}, x \in \mathbb{R} \\
\psi_{0} \text { is } 1 \text {-periodic } \\
\psi_{0}(x)>0, x \in \mathbb{R} \\
\psi_{0}(0)=1
\end{array}\right.
$$

The results of [7] show that, under our assumptions on the function $f$,

Proposition 2.1. 1) The equation (6) admits a positive and bounded solution $p>0$ if and only if $k_{0}<0$.

2) If $k_{0}<0$, the bounded solution $p>0$ of equation (6) is unique and 1-periodic in $x$.

3) Let $u_{0} \in \mathcal{C}^{0}(\mathbb{R})$ be bounded and such that $u_{0} \geq 0, u_{0} \not \equiv 0$. If $k_{0} \geq 0$, then the solution $u(t, x)$ of $(P)$ converges to 0 uniformly in $x$ as $t \rightarrow+\infty$. If $k_{0}<0$, then $u(t, x)$ converges to the solution $p>0$ of equation (6) locally uniformly in $x$, as $t \rightarrow+\infty$.

The point 3) of the above proposition shows that, whatever the initial condition, the asymptotic spreading speed of the solution $u(t, x)$ is equal to 0 if $k_{0} \geq 0$. In the remaining part of the manuscript, we need to observe positive spreading speeds. As a consequence, we have to assume that $k_{0}<0$. A sufficient condition on $r(x)$ such that $k_{0}<0$ is (see proposition 2.9 in [7]):

$$
\int_{0}^{1} r(x) d x \geq 0, \text { with } r \not \equiv 0 \text { on }[0,1] \text {. }
$$




\subsection{Initial conditions and asymptotic spreading speed}

In the case of Heaviside and compactly supported initial conditions, the asymptotic spreading speed $w^{*}$ exists, does not depend on this initial condition, is finite and satisfies (3).

Now, we consider a family of bounded front-like initial conditions $u_{0, \lambda}$ satisfying for some $\alpha \in(0,1), x_{0}>0$ and all $\lambda>0$ :

$$
u_{0, \lambda} \in \mathcal{C}^{0, \alpha}(\mathbb{R}), u_{0, \lambda} \geq 0, \liminf _{x \rightarrow-\infty} u_{0, \lambda}(x)>0,
$$

and

$$
u_{0, \lambda}(x)=e^{-\lambda x} \text { for } x \geq x_{0} .
$$

We define the asymptotic spreading speed of the solution $u_{\lambda}(t, x)$ of the Cauchy problem $(P)$ starting with the initial condition $u_{0}(x)=u_{0, \lambda}(x)$ as the nonnegative real number $w_{\lambda}$ such that:

$$
\left\{\begin{array}{l}
\lim _{t \rightarrow+\infty} u_{\lambda}(t, x+c t)=0, \forall c>w_{\lambda}, x \in \mathbb{R}, \\
\liminf _{t \rightarrow+\infty} u_{\lambda}(t, x+c t)>0, \forall 0 \leq c<w_{\lambda}, x \in \mathbb{R} .
\end{array}\right.
$$

Depending on the initial condition, the asymptotic spreading speed could be finite or not $[28,41]$. In the case of Heaviside and compactly supported initial conditions, we have already mentioned that the asymptotic spreading speed $w^{*}$ is finite and (3) is satisfied. Besides, this asymptotic spreading speed is characterised by the following formula (see [22]):

$$
w^{*}=\min _{\lambda>0} \frac{-k_{\lambda}}{\lambda},
$$

where $k_{\lambda}$ is the principal eigenvalue of the operator:

$$
L_{\lambda}: \psi_{\lambda} \mapsto-D \psi_{\lambda}^{\prime \prime}+2 \lambda D \psi_{\lambda}^{\prime}-\lambda^{2} D \psi_{\lambda}-r(x) \psi_{\lambda},
$$

with periodicity conditions, where $r(x)=\partial_{u} f(x, 0)$.

The next result describes the asymptotic spreading speed in terms of the initial condition. It is well known in the case of Heaviside and compactly supported initial conditions. To the best of our knowledge, it is not clearly stated in the existing literature for exponentially decaying initial conditions. In this proposition we show the existence of a finite asymptotic spreading speed $w_{\lambda}$ and we give a formula for $w_{\lambda}$, under our assumptions on the initial conditions $u_{0, \lambda}$.

Proposition 2.2. Assume that $f$ satisfies (1), (2), the regularity assumptions of Section 2.1 and that $k_{0}<0$ (see Section 2.2). Let $u_{0, \lambda}$ satisfy (10), (11) and $u_{\lambda}(t, x)$ be the solution of problem $(P)$ with initial condition $u_{0, \lambda}(x)$. Then, we can associate to $u_{\lambda}(t, x)$ a finite asymptotic spreading speed $w_{\lambda}$ such that

$$
\left\{\begin{array}{l}
w_{\lambda}=\frac{-k_{\lambda}}{\lambda} \text { if } 0<\lambda<\lambda^{*} \\
w_{\lambda}=w^{*} \text { otherwise, }
\end{array}\right.
$$


where $k_{\lambda}$ is the principal eigenvalue of the operator defined by (14) and $\lambda^{*}=\lambda^{*}(r)$ is the unique positive real number such that $w^{*}=\frac{-k_{\lambda^{*}}}{\lambda^{*}}$.

Proof. See the proof of this proposition in Section 4.

\subsection{Uniqueness result}

Finally we state our main uniqueness result.

Theorem 2.1. Let $f$ (resp. $\tilde{f}$ ) check (1), (2), the regularity assumptions of Section 2.1 and assume that $k_{0}<0$ (resp. $\left.\tilde{k}_{0}<0\right)$ and $r(x) \in \mathcal{M}_{1}\left(\operatorname{resp} . \partial_{u} \tilde{f}(x, 0)=\tilde{r}(x) \in \mathcal{M}_{1}\right)$. Let $u_{\lambda}(t, x)$ (resp. $\tilde{u}_{\lambda}(t, x)$ ), be the solution of the Cauchy problem $(P)$ (resp. ( $(\tilde{P})$ ) associated to $f$ and $u_{0, \lambda}(x)$ (resp. $\tilde{f}$ and $u_{0, \lambda}(x)$ ). If we assume that the asymptotic spreading speeds $w_{\lambda}$ (resp. $\tilde{w}_{\lambda}$ ) associated to $u_{\lambda}$ (resp. $\tilde{u}_{\lambda}$ ) coincide on a continuum of values of $\lambda$, that is to say

$$
\exists \lambda_{1}>0 \text { such that } \forall \lambda \in\left(0, \lambda_{1}\right), w_{\lambda}=\tilde{w}_{\lambda},
$$

then

$$
\tilde{r}(x)=r(x) \text { or } \tilde{r}(x)=r(-x+\theta) .
$$

This result shows that the linear part $r(x)=\partial_{u} f(x, 0)$ of the reaction term $f(x, u)$ in $(P)$ is uniquely determined (up to a symmetry) by the knowledge of the asymptotic spreading speeds $w_{\lambda}$, for $\lambda \in\left(0, \lambda_{1}\right)$.

The proof is based on the equality of the first and the second moments of the functions $r(x)$ and $\tilde{r}(x)$ (see Lemma 3.2 and Lemma 3.3). The derivation of the equality of the higher-order moments would be more involved, but would enable one to extend the result of Theorem 2.1 to more general classes of functions than those in $\mathcal{M}_{1}$.

\section{Proof of the main Theorem}

Our goal is to reconstruct any potential $r(x)$ element of $\mathcal{M}_{1}$, from observations of the asymptotic spreading speed which corresponds to a physical and measurable observation. Let $w_{\lambda}(r)$ and $w_{\lambda}(\tilde{r})$ be the asymptotic spreading speed associated to $r$ and $\tilde{r}$ respectively.

From the assumption of Theorem 2.1, we have $w_{\lambda}(r)=w_{\lambda}(\tilde{r})$ for all $\lambda \in\left(0, \lambda_{1}\right)$. Thus, from Proposition 2.2, $k_{\lambda}(r)=k_{\lambda}(\tilde{r})$ for all $\lambda \in\left(0, \min \left(\lambda_{1}, \lambda^{*}(r), \lambda^{*}(\tilde{r})\right)\right)$.

Lemma 3.1. For any $r \in \mathcal{M}_{1}$, the function : $\lambda \mapsto k_{\lambda}(r)$ is analytic.

This result follows from the analyticity of the coefficients in (14) and from the simplicity of $k_{\lambda}$, the principal eigenvalue of $L_{\lambda}$ (see [32]).

The analyticity of the functions $\lambda \mapsto k_{\lambda}(r)$ and $\lambda \mapsto k_{\lambda}(\tilde{r})$ implies that

$$
(H): k_{\lambda}(r)=k_{\lambda}(\tilde{r}) \text {, for all } \lambda>0 \text {. }
$$


We want to prove that the property $(\mathrm{H})$ implies that:

$$
\tilde{r}(x)=r(x) \text { or } \tilde{r}(x)=r(-x+\theta) .
$$

For this, we consider $r(x), \tilde{r}(x)$ two elements of $\mathcal{M}_{1}$ such that

$$
r(x)=a x+b \text { if } x \in[0, \theta), r(x)=c x+d \text { if } x \in[\theta, 1), \text { for } a, b, c, d \in \mathbb{R},
$$

and

$$
\tilde{r}(x)=\tilde{a} x+\tilde{b} \text { if } x \in[0, \theta), \tilde{r}(x)=\tilde{c} x+\tilde{d} \text { if } x \in[\theta, 1) \text {, for } \tilde{a}, \tilde{b}, \tilde{c}, \tilde{d} \in \mathbb{R} \text {. }
$$

Using the regularity and periodicity hypothesis of $r(x)$, we deduce

$$
\left\{\begin{array}{l}
r(x)=a x+b, \text { for } x \in[0, \theta), \\
r(x)=-\left(\frac{a \theta}{1-\theta}\right) x+b+\frac{a \theta}{1-\theta}, \text { if } x \in[\theta, 1) .
\end{array}\right.
$$

Thus, from (16), there exist $A, B \in \mathbb{R}$ such that

$$
\tilde{r}(x)=A r(x)+B, \forall x \in \mathbb{R} .
$$

The proof will be developed in several parts. In a first step we prove the lemma:

Lemma 3.2. For any $r, \tilde{r} \in \mathcal{M}_{1}$ and verifying the hypothesis $(H)$, we have:

$$
\int_{0}^{1} r(x) d x=\int_{0}^{1} \tilde{r}(x) d x=\bar{r}
$$

Proof. We consider the following problem:

$$
\left\{\begin{array}{l}
-D \psi_{\lambda}^{\prime \prime}+2 \lambda D \psi_{\lambda}^{\prime}-\left(\lambda^{2} D+r(x)\right) \psi_{\lambda}=k_{\lambda}(r) \psi_{\lambda} \quad \text { in } \quad \mathbb{R} \\
\psi_{\lambda}>0 \\
\psi_{\lambda} \text { 1-periodic } \\
\psi_{\lambda}(0)=1
\end{array}\right.
$$

According to [5] (Theorem 2.1 p.8), we have as $\lambda \rightarrow+\infty$

$$
k_{\lambda}(r)+\lambda^{2} D \rightarrow-\int_{0}^{1} r(x) d x, \text { and } k_{\lambda}(\tilde{r})+\lambda^{2} D \rightarrow-\int_{0}^{1} \tilde{r}(x) d x .
$$

Thus, using property $(\mathrm{H})$ and (19) we deduce the result of Lemma 3.2 (see another proof of this result in Lemma 5.1).

Lemma 3.3. For any $r, \tilde{r} \in \mathcal{M}_{1}$, and verifying the property (H), we have:

$$
\int_{0}^{1}(r(x)-\bar{r})^{2} d x=\int_{0}^{1}(\tilde{r}(x)-\bar{r})^{2} d x .
$$


Proof. See the proof of this Lemma in Section 5.

From (17) we obtain

$$
\int_{0}^{1} \tilde{r}^{2}(x) d x=A^{2} \int_{0}^{1} r^{2}(x) d x+2 A B \int_{0}^{1} r(x) d x+B^{2},
$$

by using Lemma 3.2 and Lemma 3.3 we get

$$
\left(1-A^{2}\right) \int_{0}^{1} r^{2}(x) d x=B\left((1+A) \int_{0}^{1} r(x) d x\right),
$$

for $A \neq-1$ we obtain

$$
(1-A) \int_{0}^{1} r^{2}(x) d x=B \int_{0}^{1} r(x) d x
$$

by using the fact that $B=(1-A) \int_{0}^{1} r(x) d x$, for $A \neq 1$, we conclude that

$$
\int_{0}^{1} r^{2}(x) d x=\left(\int_{0}^{1} r(x) d x\right)^{2}
$$

From the Cauchy inequality, the equality (20) is true if and only if $r$ is constant. Thanks to (17) we deduce that $\tilde{r}$ is also constant, and according to Lemma 3.2 we obtain $r=\tilde{r}$. By symmetry we also deduce that if $\tilde{r}$ is constant then $r$ is constant so $\tilde{r}=r$.

Now we consider the case $A=1$. We have

$$
\int_{0}^{1} \tilde{r}(x) d x=\int_{0}^{1} r(x) d x+B
$$

By using the fact that $\int_{0}^{1} r(x) d x=\int_{0}^{1} \tilde{r}(x) d x$, we conclude that $B=0$, so $r(x)=\tilde{r}(x)$. Finally, if $A=-1$, then $\tilde{r}(x)=-r(x)+B$, so $\tilde{r}(x)=r(-x+\theta)$.

Remark 1. In the homogeneous case, i.e, if $f$ does not depend on $x$, the uniqueness result of Theorem 2.1 is obvious. In such case, it is known [2, 34] that case $w^{*}=2 \sqrt{r D}$, thus $w^{*}=\tilde{w}^{*}$ implies that $r=\tilde{r}$.

\section{Proof of Proposition 2.2}

Recall that for the Cauchy problem $(P)$ associated with compactly supported or Heaviside initial conditions, we can define an asymptotic speed of propagation (see formula (3)). In the case of exponentially decaying initial conditions of the type (10)(11) we have to prove the existence of this speed.

The proof is based on the construction of appropriate sub and super-solutions for the system $(P)$. We are going to use the notion of pulsating traveling wave for the equation:

$$
\partial_{t} u=D \partial_{x x} u+g_{l}(x, u)
$$


with $g_{l}(x, u)=\frac{1}{l} f(x, l u)$ for some $l>0$. Note that,

$$
\partial_{u} g_{l}(x, 0)=\partial_{u} f(x, 0), \forall l>0 .
$$

In view to carry out sub-solutions and super-solutions of problem $(P)$, we consider the following problem:

$$
\left\{\begin{array}{l}
-D \partial_{x x} p_{l}=g_{l}\left(x, p_{l}\right), x \in \mathbb{R} \\
p_{l} \text { is 1-periodic } \\
p_{l}>0
\end{array}\right.
$$

Now, we consider the solution $u_{\lambda}(t, x)$ of $(P)$ with initial condition $u_{0, \lambda}(x)$. We construct a super-solution for this problem. In that respect we first look for $l$ such that the solution $p_{l}(x)$ of $(21)$ satisfies:

$$
\min _{\mathbb{R}} p_{l}>\sup _{\mathbb{R}} u_{0, \lambda}
$$

Let us show that such a value of $l$ exists. Let $p(x)=p_{1}(x)$ be the unique positive solution of

$$
-D \partial_{x x} p=f(x, p), x \in \mathbb{R} \text {. }
$$

It is known, from Proposition 2.1, that $p(x)$ is strictly positive and periodic. Thus, it exists $\delta_{1}, \delta_{2}>0$ such that

$$
0<\delta_{1}<p(x)<\delta_{2} \text { on } \mathbb{R} \text {. }
$$

Recall that the function $p_{l}$ satisfies: $-D \partial_{x x} p_{l}=\frac{1}{l} f\left(x, l p_{l}\right)$ for $x \in \mathbb{R}$. Let us set $q(x)=l p_{l}(x)$. Then $q$ verifies the following equation:

$$
-D \partial_{x x} q=f(x, q)
$$

By uniqueness (Proposition 2.1), we deduce $q(x)=p(x)$, and finally $p_{l}(x)=\frac{p(x)}{l}$. From (24) we obtain:

$$
\frac{\delta_{1}}{l}<p_{l}(x)<\frac{\delta_{2}}{l}
$$

Let $l_{1}<1$ be such that $\frac{\delta_{1}}{l_{1}}>\sup _{\mathbb{R}} u_{0, \lambda}$. Then we can write

$$
\min _{\mathbb{R}} p_{l_{1}}>\sup _{\mathbb{R}} u_{0, \lambda}
$$

In a first step we consider $\lambda^{\prime} \in(0, \lambda)$ such that $\lambda^{\prime}<\lambda^{*}$ and let $c^{\prime}=\frac{-k_{\lambda^{\prime}}}{\lambda^{\prime}}>c^{*}=\frac{-k_{\lambda^{*}}}{\lambda^{*}}$. We consider $U_{c^{\prime}}^{l_{1}}(t, x)$, a pulsating traveling wave solution of the problem:

$$
\left\{\begin{array}{l}
\partial_{t} U_{c^{\prime}}^{l_{1}}=D \partial_{x x} U_{c^{\prime}}^{l_{1}}+g_{l_{1}}\left(x, U_{c^{\prime}}^{l_{1}}\right), t \in \mathbb{R}, x \in \mathbb{R}, \\
\forall z \in \mathbb{Z}, \forall(t, x) \in \mathbb{R} \times \mathbb{R}, U_{c^{\prime}}^{l_{1}}\left(t+\frac{z}{c^{\prime}}, x\right)=U_{c^{\prime}}^{l_{1}}(t, x-z), \\
\forall(t, x) \in \mathbb{R} \times \mathbb{R}, 0 \leq U_{c^{\prime}}^{l_{1}}(t, x) \leq p_{l_{1}}(x), \\
\lim _{x \rightarrow-\infty}\left|U_{c^{\prime}}^{l_{1}}(t, x)-p_{l_{1}}(x)\right|=0 \text { and } \lim _{x \rightarrow+\infty} U_{c^{\prime}}^{l_{1}}(t, x)=0,
\end{array}\right.
$$


where the above limits hold locally in $t$. The existence of this solution follows from [8] and its uniqueness (up to a translation) is proved in [29].

From [25], it is known that the asymptotic behavior of $U_{c^{\prime}}^{l_{1}}(t, x)$ is:

$$
U_{c^{\prime}}^{l_{1}}(t, x) \sim B e^{-\lambda_{c^{\prime}}\left(x-c^{\prime} t\right)} \psi_{\lambda_{c^{\prime}}}(x), \text { as } x \rightarrow+\infty, \text { for } B>0, t \in \mathbb{R},
$$

where $\lambda_{c^{\prime}}$ is such that

$$
\lambda_{c^{\prime}}=\inf \left\{\lambda>0 \text { such that } k_{\lambda}+\lambda c^{\prime}=0\right\},
$$

and $\psi_{\lambda_{c^{\prime}}}(x)$ is the eigenfunction of the operator $L_{\lambda_{c^{\prime}}}$ (see (14)) associated to the principal eigenvalue $k_{\lambda_{c^{\prime}}}$. From the convexity of the map $\lambda \mapsto-k_{\lambda}$ (see [7]) and by definition of $\lambda^{*}$ (see Proposition 2.2), the equation $k_{\lambda}=-\lambda c^{\prime}$ admits at most two roots, $\lambda^{-} \leq \lambda^{*} \leq \lambda^{+}$. Since $\lambda^{\prime}<\lambda^{*}$, we get that $\lambda^{\prime}=\lambda^{-}=\lambda_{c^{\prime}}$.

Moreover, since $l_{1}<1$ and $\frac{f(x, s)}{s}$ is decreasing we have

$$
\frac{f\left(x, l_{1} s\right)}{l_{1} s}>\frac{f(x, s)}{s} .
$$

Thus $g_{l_{1}}=\frac{1}{l_{1}} f\left(x, l_{1} s\right)>f(x, s)$ for all $s \geq 0$.

Finally $U_{c^{\prime}}^{l_{1}}(t, x)$ satisfies

$$
\partial_{t} U_{c^{\prime}}^{l_{1}}-D \partial_{x x} U_{c^{\prime}}^{l_{1}}=g_{l_{1}}\left(x, U_{c^{\prime}}^{l_{1}}\right)>f\left(x, U_{c^{\prime}}^{l_{1}}\right), x \in \mathbb{R} .
$$

Then $U_{c^{\prime}}^{l_{1}}(t, x)$ is a super-solution for the problem $(P)$.

Now we want to prove that

$$
U_{c^{\prime}}^{l_{1}}(0, x) \geq u_{0, \lambda}(x), \forall x \in \mathbb{R} .
$$

From (28), we get

$$
U_{c^{\prime}}^{l_{1}}(0, x) \sim B e^{-\lambda^{\prime} x} \psi_{\lambda^{\prime}}(x), \text { as } x \rightarrow+\infty .
$$

Using that $\psi_{\lambda^{\prime}}(x)$ is continuous, 1 -periodic, $\psi_{\lambda^{\prime}}(x)$ strictly positive on $[0,1]$ and $\lambda^{\prime}<\lambda$, we obtain

$$
B e^{-\lambda^{\prime} x} \psi_{\lambda^{\prime}}(x)>e^{-\lambda x} \text {, for } x \text { large enough. }
$$

Then, there exists $M>0$ such that for any $x>M$,

$$
U_{c^{\prime}}^{l_{1}}(0, x) \geq e^{-\lambda x}=u_{0, \lambda}(x) .
$$

From (26) and (27), we deduce that there exists $m<0$ such that

$$
\min _{x \leq m} U_{c^{\prime}}^{l_{1}}(0, x) \geq \sup _{x \in \mathbb{R}} u_{0, \lambda}(x) .
$$

It remains to prove that

$$
\min _{x \in[m, M]} U_{c^{\prime}}^{l_{1}}(0, x) \geq \sup _{x \in \mathbb{R}} u_{0, \lambda}(x)
$$

Let

$$
\delta=\min _{x \in[m, M]} U_{c^{\prime}}^{l_{1}}(0, x)
$$


From the strong parabolic maximum principle, we know that $\delta>0$. Let $L \geq m$ be large enough such that

$$
e^{-\lambda x}<\delta \text {, for all } x>L
$$

Then $U_{c^{\prime}}^{l_{1}}(0, x-L+m)$ verifies (30). Indeed:

- If $x \leq L$, then $x-L+m \leq m$. From (33), it follows that

$$
U_{c^{\prime}}^{l_{1}}(0, x-L+m) \geq \sup _{x \in \mathbb{R}} u_{0, \lambda}(x) .
$$

- If $x \in(L, L+M-m)$, then $m \leq x-L+m \leq M$

and, from (34),

$$
U_{c^{\prime}}^{l_{1}}(0, x-L+m)>\delta>e^{-\lambda x}=u_{0, \lambda}(x) .
$$

- If $x \geq L+M-m$, then $x-L+m \geq M$

and, from (32),

$$
U_{c^{\prime}}^{l_{1}}(0, x-L+m) \geq e^{-\lambda(x-L+m)} \geq e^{-\lambda x} e^{+\lambda(L-m)} \geq e^{-\lambda x}=u_{0, \lambda}(x) .
$$

Thus, even if it means translating $U_{c^{\prime}}^{l_{1}}(0, x)$ to the right, we can assume that $U_{c^{\prime}}^{l_{1}}(0, x)>$ $u_{0, \lambda}(x), \forall x \in \mathbb{R}$. Since $U_{c^{\prime}}^{l_{1}}(0, x)>u_{0, \lambda}(x)$ and since $U_{c^{\prime}}^{l_{1}}$ is a super-solution of $(P)$, a comparison principle implies that

$$
\text { for all } \lambda^{\prime} \in\left(0, \min \left(\lambda, \lambda^{*}\right)\right), U_{c^{\prime}}^{l_{1}}(t, x)>u_{\lambda}(t, x), t \geq 0, x \in \mathbb{R},
$$

with $c^{\prime}=\frac{-k_{\lambda^{\prime}}}{\lambda^{\prime}}$.

In a second step we consider $\lambda^{\prime \prime}$ such that $0<\lambda<\lambda^{\prime \prime}<\lambda^{*}$ and let $c^{\prime \prime}=\frac{-k_{\lambda^{\prime \prime}}}{\lambda^{\prime \prime}}$. Let $l_{2}>1$ be such that $\frac{\delta_{2}}{l_{2}}<\liminf _{x \rightarrow-\infty} u_{0, \lambda}(x)$ (see $\left.(25)\right)$. We consider $U_{c^{\prime \prime}}^{l_{2}}(t, x)$ the pulsating traveling wave solution of the problem (27) (with $l_{2}$ instead of $l_{1}$ and $c^{\prime \prime}$ instead of $c^{\prime}$ ). Using the same arguments as above, and the fact that $\max _{\mathbb{R}} p_{l_{2}}<\frac{\delta_{2}}{l_{2}}<\liminf _{x \rightarrow-\infty} u_{0, \lambda}(x)$, it is easily seen that $U_{c^{\prime \prime}}^{l_{2}}(t, x)$ is a sub-solution of the problem $(P)$ satisfying

$$
U_{c^{\prime \prime}}^{l_{2}}(0, x)<u_{0, \lambda}(x)
$$

(even if it means translating $U_{c^{\prime \prime}}^{l_{2}}(0, x)$ to the left). A comparison principle implies that

$$
\text { for all } \lambda^{\prime \prime} \in\left(\lambda, \lambda^{*}\right), U_{c^{\prime \prime}}^{l_{2}}(t, x)<u_{\lambda}(x), t \geq 0, x \in \mathbb{R} \text {. }
$$

So we get from (35) and (36) an appropriate pair of sub and super-solutions for the problem $(P)$, and we can write:

$$
U_{c^{\prime \prime}}^{l_{2}}(t, x)<u_{\lambda}(t, x)<U_{c^{\prime}}^{l_{1}}(t, x) \text {, for all } t \geq 0, x \in \mathbb{R}, \lambda \in\left(\lambda^{\prime}, \lambda^{\prime \prime}\right) .
$$

Now we are going to complete the proof of the existence of an asymptotic spreading speed associated to problem $(P)$ with initial conditions of the type (10)-(11).

- First case: if $\lambda<\lambda^{*}$, we prove that $w_{\lambda}=\frac{-k_{\lambda}}{\lambda}$ verifies (12). 
(i) Let $c>w_{\lambda}=\frac{-k_{\lambda}}{\lambda}$, we want to prove that

$$
\lim _{t \rightarrow+\infty} u_{\lambda}(t, x+c t)=0, \text { for all } x \in \mathbb{R} \text {. }
$$

Let $\lambda^{\prime} \in(0, \lambda)$ be such that $\frac{-k_{\lambda}}{\lambda}<\frac{-k_{\lambda^{\prime}}}{\lambda^{\prime}}<c$; the existence of $\lambda^{\prime}$ follows from the continuity of $s \mapsto \frac{k_{s}}{s}$. We know from $(35)$ that $u_{\lambda}(t, x+c t)<U_{c^{\prime}}^{l_{1}}(t, x+c t)$, with $w_{\lambda}<c^{\prime}=\frac{-k_{\lambda^{\prime}}}{\lambda^{\prime}}<c$. Since $c^{\prime}<c$ we obtain from $(27) \lim _{t \rightarrow+\infty} U_{c^{\prime}}^{l_{1}}(t, x+c t)=$ 0 . We conclude that if $c>w_{\lambda}$ then

$$
\lim _{t \rightarrow+\infty} u_{\lambda}(t, x+c t)=0, \text { for all } x \in \mathbb{R} .
$$

(ii) Let $c<w_{\lambda}=\frac{-k_{\lambda}}{\lambda}$. We want to prove that

$$
\liminf _{t \rightarrow+\infty} u_{\lambda}(t, x+c t)>0
$$

Let $\lambda^{\prime \prime} \in\left(\lambda, \lambda^{*}\right)$ be such that $c<\frac{-k_{\lambda^{\prime \prime}}}{\lambda^{\prime \prime}}<\frac{-k_{\lambda}}{\lambda}$. We know from (36), that $U_{c^{\prime \prime}}^{l_{2}}(t, x+c t)<u_{\lambda}(t, x+c t)$, with $c<c^{\prime \prime}=\frac{-k_{\lambda^{\prime \prime}}}{\lambda^{\prime \prime}}<w_{\lambda}$. Since $c<c^{\prime \prime}$ we obtain from (27) that $\liminf _{t \rightarrow+\infty} U_{c^{\prime \prime}}^{l_{2}}(t, x+c t)>0$. We conclude that if $c<w_{\lambda}$ then

$$
\liminf _{t \rightarrow+\infty} u_{\lambda}(t, x+c t)>0, \text { for all } x \in \mathbb{R}
$$

Using (37) together with (38) imply that $w_{\lambda}=\frac{-k_{\lambda}}{\lambda}$ is the spreading speed of $u_{\lambda}$ in the sense of $(12)$ when $\lambda \in\left(0, \lambda^{*}\right)$.

- Second case: if $\lambda \geq \lambda^{*}$, we prove that $w_{\lambda}=w^{*}$.

(i) Let $c>w^{*}$, and consider $\lambda^{\prime} \in\left(0, \lambda^{*}\right)$ such that $w^{*}<\frac{-k_{\lambda^{\prime}}}{\lambda^{\prime}}=c^{\prime}<c$. From (35), we know that

$$
0<u_{\lambda}(t, x)<U_{c^{\prime}}^{l_{1}}(t, x), t \geq 0, x \in \mathbb{R} .
$$

From (3) and since $0<c^{\prime}<c$, it follows that

$$
\lim _{t \rightarrow+\infty} u_{\lambda}(t, x+c t)=0, \text { for all } x \in \mathbb{R} \text {. }
$$

(ii) Let $c<w^{*}$ and $V(t, x)$ be a solution of $(P)$ with compactly supported initial condition such that $0 \leq V(0, x)<u_{0, \lambda}(x)$ for all $x \in \mathbb{R}$. A comparison principle implies that

$$
0<V(t, x)<u_{\lambda}(t, x) \text { for all } t>0, x \in \mathbb{R} \text {. }
$$

Thus

$$
u_{\lambda}(t, x+c t)>V(t, x+c t), \text { for all } t>0, x \in \mathbb{R} .
$$

From (27), we know that

$$
\liminf _{t \rightarrow+\infty} V(t, x+c t)>0, \text { for all } x \in \mathbb{R} \text {. }
$$


Coefficient determination via asymptotic spreading speeds

Thus

$$
\liminf _{t \rightarrow+\infty} u_{\lambda}(t, x+c t)>0, \text { for all } x \in \mathbb{R} .
$$

Using (39) together with (40) imply that $w^{*}$ is the spreading speed of $u_{\lambda}$ for all $\lambda \geq \lambda^{*}$.

\section{Proof of Lemma 3.3}

We consider the following problem

$$
\left\{\begin{array}{l}
D \psi_{\lambda}^{\prime \prime}-2 \lambda D \psi_{\lambda}^{\prime}+r(x) \psi_{\lambda}=-l_{\lambda}(r) \psi_{\lambda} \text { in } \mathbb{R} \\
\psi_{\lambda}>0 \\
\psi_{\lambda} \text { 1-periodic } \\
\psi_{\lambda}(0)=1
\end{array}\right.
$$

where $l_{\lambda}(r)=k_{\lambda}(r)+\lambda^{2} D$. We define the functions $r_{\lambda}$ and $h_{\lambda}$ by

$$
l_{\lambda}(r)=-\bar{r}+\frac{r_{\lambda}}{\lambda^{2}}
$$

and

$$
\psi_{\lambda}(x)=1+\frac{f_{1}(x)}{\lambda}+\frac{h_{\lambda}(x)}{\lambda^{2}}
$$

where

$$
f_{1}(x):=\frac{1}{2 D} \int_{0}^{x}(r(s)-\bar{r}) d s .
$$

We derive from (41):

$$
\begin{gathered}
0=\left(2 D f_{1}^{\prime}-r(x)-l_{\lambda}(r)\right)+\frac{1}{\lambda}\left(-D f_{1}^{\prime \prime}+2 D h_{\lambda}^{\prime}-r(x) f_{1}-l_{\lambda}(r) f_{1}\right) \\
+\frac{1}{\lambda^{2}}\left(-D h_{\lambda}^{\prime \prime}-r(x) h_{\lambda}-l_{\lambda}(r) h_{\lambda}\right) \\
=\left(-l_{\lambda}(r)-\bar{r}\right)+\frac{1}{\lambda}\left(-D f_{1}^{\prime \prime}+2 D h_{\lambda}^{\prime}-r(x) f_{1}+\bar{r} f_{1}\right) \\
+\frac{1}{\lambda^{2}}\left(-D h_{\lambda}^{\prime \prime}-r(x) h_{\lambda}+\bar{r} h_{\lambda}\right)-\frac{1}{\lambda^{3}} r_{\lambda} f_{1}-\frac{1}{\lambda^{4}} r_{\lambda} h_{\lambda} \\
=\frac{1}{\lambda}\left(-D f_{1}^{\prime \prime}+2 D h_{\lambda}^{\prime}-r(x) f_{1}+\bar{r} f_{1}\right) \\
+\frac{1}{\lambda^{2}}\left(-D h_{\lambda}^{\prime \prime}-r(x) h_{\lambda}+\bar{r} h_{\lambda}-r_{\lambda}\right)-\frac{1}{\lambda^{3}} r_{\lambda} f_{1}-\frac{1}{\lambda^{4}} r_{\lambda} h_{\lambda} .
\end{gathered}
$$

Now we prove that $a_{2}:=\lim _{\lambda \rightarrow+\infty} r_{\lambda}$ and $f_{2}(x):=\lim _{\lambda \rightarrow+\infty} h_{\lambda}(x)$ are well-defined by using Lemma 5.1.

Lemma 5.1. There exists a constant $C>0$ such that

$$
\left|l_{\lambda}(r)+\bar{r}\right| \leq C / \lambda
$$


Coefficient determination via asymptotic spreading speeds

Proof. We know (see [7] for example) that

$$
\begin{aligned}
l_{\lambda}(r) & =\sup \left\{l \in \mathbb{R}, \quad \exists \psi \in W_{\text {per }}^{2, \infty}(\mathbb{R}), \quad \psi>0 \text { in } \mathbb{R}, \quad-L_{\lambda} \psi \geq l \psi \text { a.e. in } \mathbb{R}\right\}, \\
& =\inf \left\{l \in \mathbb{R}, \quad \exists \psi \in W_{\text {per }}^{2, \infty}(\mathbb{R}), \quad \psi>0 \text { in } \mathbb{R}, \quad-L_{\lambda} \psi \leq l \psi \text { a.e. in } \mathbb{R}\right\} .
\end{aligned}
$$

Using $\psi(x)=1+\frac{f_{1}(x)}{\lambda}$ as a test-function, taking $\lambda$ large enough so that $1+\frac{f_{1}(x)}{\lambda}>0$ for all $x$, we get almost everywhere:

$$
\begin{aligned}
-L_{\lambda} \psi & =-\frac{D f_{1}^{\prime \prime}(x)}{\lambda}+\frac{2 \lambda D f_{1}^{\prime}(x)}{\lambda}-r(x)-\frac{r(x) f_{1}(x)}{\lambda}, \\
& =-\frac{r^{\prime}(x)}{2 \lambda}+r(x)-\bar{r}-r(x)-\frac{r(x) f_{1}(x)}{\lambda}, \\
& \geq-\frac{C_{0}}{\lambda}-\bar{r}
\end{aligned}
$$

where $C_{0}$ is a constant which only depends on $\|r\|_{\infty}$ and $\left\|r^{\prime}\right\|_{\infty}$. Take $C$ large enough so that

$$
C+\frac{C f_{1}(x)}{\lambda}+\bar{r} f_{1}(x) \geq C_{0} .
$$

We derive from this inequality that

$$
\left(-\frac{C}{\lambda}-\bar{r}\right) \psi \leq-\frac{C_{0}}{\lambda}-\bar{r} \quad \text { over } \mathbb{R} .
$$

Hence $-L_{\lambda} \psi \geq\left(-\frac{C}{\lambda}-\bar{r}\right) \psi$ over $\mathbb{R}$ and we derive from the definition of $l_{\lambda}(r)$ that

$$
l_{\lambda}(r) \geq-\frac{C}{\lambda}-\bar{r} .
$$

Similarly, one can prove that, taking $C$ larger if necessary,

$$
l_{\lambda}(r) \leq \frac{C}{\lambda}-\bar{r} .
$$

Define $\theta_{\lambda} \in W_{\text {per }}^{2, \infty}(\mathbb{R})$ so that

$$
\psi_{\lambda}(x)=1+\frac{f_{1}(x)}{\lambda}+\frac{\theta_{\lambda}(x)}{\lambda},
$$

where

$$
f_{1}(x):=\frac{1}{2 D} \int_{0}^{x}(r(s)-\bar{r}) d s .
$$

Rewriting the identity $-L_{\lambda} \psi_{\lambda}=l_{\lambda}(r) \psi_{\lambda}$ with this change of functions, we get:

$$
-L_{\lambda} \theta_{\lambda}+\left(\bar{r}-\frac{r_{\lambda}}{\lambda^{2}}\right) \theta_{\lambda}=D f_{1}^{\prime \prime}(x)+\left(r(x)-\bar{r}+\frac{r_{\lambda}}{\lambda^{2}}\right) f_{1}(x)+\frac{r_{\lambda}}{\lambda}=: F_{\lambda} .
$$


Lemma 5.1 yields that $\left|\frac{r_{\lambda}}{\lambda}\right| \leq C$ for all $\lambda$. Thus, $F_{\lambda} \in L_{p e r}^{\infty}(\mathbb{R})$ and there exists two constants $\tilde{\lambda}>0, C_{0}>0$, so that for all $\lambda \geq \tilde{\lambda},\left\|F_{\lambda}\right\|_{\infty} \leq C_{0}$.

Multiplying (43) by $\theta_{\lambda}^{\prime \prime}$ and integrating, one gets, for all $\lambda \geq \tilde{\lambda}$

$$
\begin{aligned}
& \int_{0}^{1}\left(\theta_{\lambda}^{\prime \prime}\right)^{2} d x=-\int_{0}^{1}\left(r(x)-\bar{r}+\frac{r_{\lambda}}{\lambda^{2}}\right) \theta_{\lambda} \theta_{\lambda}^{\prime \prime} d x-\int_{0}^{1} F_{\lambda} \theta_{\lambda}^{\prime \prime} d x, \\
= & \int_{0}^{1} r^{\prime}(x) \theta_{\lambda} \theta_{\lambda}^{\prime} d x+\int_{0}^{1}\left(r(x)-\bar{r}+\frac{r_{\lambda}}{\lambda^{2}}\right)\left(\theta_{\lambda}^{\prime}\right)^{2} d x-\int_{0}^{1} F_{\lambda} \theta_{\lambda}^{\prime \prime} d x, \\
\leq & C_{1}\left(\left\|\theta_{\lambda}^{\prime}\right\|_{L^{2}(0,1)}^{2}+\left\|\theta_{\lambda}^{\prime \prime}\right\|_{L^{2}(0,1)}\right),
\end{aligned}
$$

where $C_{1}$ is a constant which only depends on $\left\|r^{\prime}\right\|_{\infty},\|r\|_{\infty}$, the constant $C$ given by Lemma 5.1 and $C_{0}$, and where we have used the inequalities

$$
\left\|\theta_{\lambda}\right\|_{L^{2}(0,1)} \leq\left\|\theta_{\lambda}\right\|_{\infty} \leq\left\|\theta_{\lambda}^{\prime}\right\|_{L^{1}(0,1)} \leq\left\|\theta_{\lambda}^{\prime}\right\|_{L^{2}(0,1)}
$$

since $\theta_{\lambda}(0)=0$.

Assume now that there exists a sequence $\left(\lambda_{n}\right)_{n}$ such that $\lambda_{n} \geq \tilde{\lambda}$

and $\left\|\theta_{\lambda_{n}}^{\prime}\right\|_{L^{2}(0,1)} \rightarrow+\infty$ as $n \rightarrow+\infty$. Let $\zeta_{n}:=\frac{\theta_{\lambda_{n}}}{\left\|\theta_{\lambda_{n}}^{\prime}\right\|_{L^{2}(0,1)}}$, so that $\left\|\zeta_{n}^{\prime}\right\|_{L^{2}(0,1)}=1$. Dividing (44) by $\left\|\theta_{\lambda_{n}}^{\prime}\right\|_{L^{2}(0,1)}^{2}$, one gets

$$
\int_{0}^{1}\left(\zeta_{n}^{\prime \prime}\right)^{2} d x \leq C_{1}\left(1+\frac{\left\|\zeta_{n}^{\prime \prime}\right\|_{L^{2}(0,1)}}{\left\|\theta_{\lambda_{n}}^{\prime}\right\|_{L^{2}(0,1)}}\right)
$$

Thus, as $\lim _{n \rightarrow+\infty}\left\|\theta_{\lambda_{n}}^{\prime}\right\|_{L^{2}(0,1)}=+\infty,\left(\left\|\zeta_{n}^{\prime \prime}\right\|_{L^{2}(0,1)}\right)_{n}$ is bounded. As $\left\|\zeta_{n}^{\prime}\right\|_{L^{2}(0,1)}=1$ and $\left\|\zeta_{n}\right\|_{L^{2}(0,1)} \leq 1$ for all $n$ due to (45), we can extract a subsequence, that we still denote $\left(\zeta_{n}\right)_{n}$, which converges weakly in $H_{p e r}^{2}(0,1)$ as $n \rightarrow+\infty$. On the other hand, equation (43) gives

$$
2 \lambda_{n} \zeta_{n}^{\prime}=\zeta_{n}^{\prime \prime}+\left(r(x)-\bar{r}+\frac{r_{\lambda_{n}}}{\lambda_{n}^{2}}\right) \zeta_{n}+\frac{F_{\lambda_{n}}}{\left\|\theta_{\lambda_{n}}^{\prime}\right\|_{L^{2}(0,1)}}
$$

and thus the sequence $\left(\lambda_{n} \zeta_{n}^{\prime}\right)_{n}$ is bounded in $L^{2}(0,1)$, which is a contradiction since $\left\|\zeta_{n}^{\prime}\right\|_{L^{2}(0,1)}=1$ for all $n$. We thus conclude that the family $\left(\theta_{\lambda}^{\prime}\right)_{\lambda \geq \tilde{\lambda}}$ is bounded in $L^{2}(0,1)$.

It follows that $\left(\theta_{\lambda}\right)_{\lambda \geq \tilde{\lambda}}$ and $\left(\theta_{\lambda}^{\prime \prime}\right)_{\lambda \geq \tilde{\lambda}}$ are bounded in $L^{2}(0,1)$ due to (44) and (45) respectively, and thus (43) implies that $\left(\lambda \theta_{\lambda}^{\prime}\right)_{\lambda \geq \tilde{\lambda}}$ is bounded in $L^{2}(0,1)$. The same chain of inequalities yields that $\left(\lambda \theta_{\lambda}\right)_{\lambda \geq \tilde{\lambda}}$ is bounded in $H^{2}(0,1)$.

By replacing in (43) $\theta_{\lambda}$ by $\frac{h_{\lambda}}{\lambda}$, we get

$$
0=-D f_{1}^{\prime \prime}+2 D h_{\lambda}^{\prime}+(\bar{r}-r) f_{1}-\frac{1}{\lambda}\left(D h_{\lambda}^{\prime \prime}+(r-\bar{r}) h_{\lambda}+r_{\lambda}\right)-\frac{r_{\lambda} f_{1}}{\lambda^{2}}-\frac{r_{\lambda} h_{\lambda}}{\lambda^{3}} \cdot(
$$

Integrating and noticing that $\int_{0}^{1}(\bar{r}-r(x)) f_{1} d x=2 D \int_{0}^{1} f_{1} f_{1}^{\prime} d x=D \int_{0}^{1}\left(f_{1}^{2}\right)^{\prime} d x=0$, one gets

$$
0=\frac{1}{\lambda} \int_{0}^{1}(r(x)-\bar{r}) h_{\lambda} d x+\frac{r_{\lambda}}{\lambda}+\frac{r_{\lambda}}{\lambda^{2}} \int_{0}^{1} f_{1} d x+\frac{r_{\lambda}}{\lambda^{3}} \int_{0}^{1} h_{\lambda} d x
$$


and thus

$$
r_{\lambda}=-\int_{0}^{1}(r(x)-\bar{r}) h_{\lambda}(x) d x-\frac{r_{\lambda}}{\lambda} \int_{0}^{1} f_{1} d x-\frac{r_{\lambda}}{\lambda^{2}} \int_{0}^{1} h_{\lambda} d x .
$$

We have shown previously that $r_{\lambda}$ and $\left\|h_{\lambda}\right\|_{H^{2}(0,1)}$ are bounded uniformly with respect to $\lambda \geq \tilde{\lambda}$. Then $\frac{r_{\lambda}}{\lambda} \rightarrow 0$ as $\lambda \rightarrow+\infty$. Passing to the limit in (46), we get that $f_{2}:=\lim _{\lambda \rightarrow+\infty} h_{\lambda}$ is well-defined in $H^{1}(0,1)$ and that

$$
f_{2}^{\prime}=\frac{1}{2}\left(f_{1}^{\prime \prime}+\frac{(r(x)-\bar{r})}{D} f_{1}\right)
$$

Lastly, letting $\lambda \rightarrow+\infty$ in (47), we obtain

$$
\lim _{\lambda \rightarrow+\infty} r_{\lambda}=-\int_{0}^{1}(r(x)-\bar{r}) f_{2} d x=a_{2} .
$$

Now, we can claim that $a_{2}:=\lim _{\lambda \rightarrow+\infty} r_{\lambda}$ and $f_{2}(x):=\lim _{\lambda \rightarrow+\infty} h_{\lambda}(x)$ are well-defined. By taking the limit in (46) we get:

$$
-D f_{1}^{\prime \prime}+2 D f_{2}^{\prime}-r(x) f_{1}+\bar{r} f_{1}=0
$$

and

$$
a_{2}=\int_{0}^{1}(\bar{r}-r(x)) f_{2}(x) d x
$$

Define $M(x):=\int_{0}^{x} r(y) d y$ and integrate by parts:

$$
a_{2}=-\int_{0}^{1}(\bar{r} x-M(x)) f_{2}^{\prime}(x) d x
$$

(there is no boundary terms since $M(1)=\int_{0}^{1} r d x=\bar{r}$ ). We compute $f_{2}^{\prime}$ using the first equation in (48):

$$
\begin{aligned}
a_{2} & =\frac{1}{2 D} \int_{0}^{1}(M(x)-\bar{r} x)\left(D f_{1}^{\prime \prime}+(r(x)-\bar{r}) f_{1}\right) d x \\
& =-\frac{1}{2} \int_{0}^{1}(r(x)-\bar{r}) f_{1}^{\prime}(x) d x+\frac{1}{4 D} \int_{0}^{1} \frac{d}{d x}(M(x)-\bar{r} x)^{2} f_{1}(x) d x \\
& =-\frac{1}{2} \int_{0}^{1}(r(x)-\bar{r}) f_{1}^{\prime}(x) d x-\frac{1}{4 D} \int_{0}^{1}(M(x)-\bar{r} x)^{2} f_{1}^{\prime}(x) d x \\
& =-\frac{1}{4 D} \int_{0}^{1}(r(x)-\bar{r})^{2} d x-\frac{1}{24 D^{2}} \int_{0}^{1} \frac{d}{d x}(M(x)-\bar{r} x)^{3} d x \\
& =-\frac{1}{4 D} \int_{0}^{1}(r(x)-\bar{r})^{2} d x .
\end{aligned}
$$


Hence, we conclude that

$$
l_{\lambda}(r)=-\bar{r}-\frac{1}{4 D \lambda^{2}} \int_{0}^{1}(r(x)-\bar{r})^{2} d x+o\left(\frac{1}{\lambda^{2}}\right)
$$

from which the conclusion follows from Lemma 3.2 and $l_{\lambda}(r)=l_{\lambda}(\tilde{r})$ for all $\lambda>0$.

\section{Acknowledgments}

This work was partially supported by the franco-algerian agreement Tassili 11 MDU 834 (second author).

\section{References}

[1] Alifanov O M, Artyukhin E A and Rumyantsev S V 1996 Extreme Methods for Solving Ill-Posed Problems with Application to Inverse Heat Transfer Problems. (New York: Begell House Inc.)

[2] Aronson D G and Weinberger H G 1975 Nonlinear diffusion in population genetics, combustion and nerve propagation Partial Differential Equations and Related Topics Lectures Notes in Mathematics (New York: Springer) 446 5-49.

[3] Aronson D G and Weinberger H G 1978 Multidimensional nonlinear diffusion arising in population genetics Adv. Math. 30 33-76.

[4] Berestycki H and Hamel F 2002 Front propagation in periodic excitable media Comm Pure Appl Math 558 949-1032.

[5] Berestycki H, Hamel F and Nadirashvili N 2005 Elliptic eigenvalue problems with large drift and applications to nonlinear propagation phenomena Comm in Mathematical Physics 253 451-480.

[6] Berestycki H, Hamel F, and Nadin G 2008 Asymptotic spreading in heterogeneous diffusive excitable media J Funct Anal 2559 2146-2189.

[7] Berestycki H, Hamel F and Roques L 2005 Analysis of the periodically fragmented environment model: I- Species persistence J. Math. Biol. 51 (1) 75-113.

[8] Berestycki H, Hamel F, and Roques L 2005 Analysis of the periodically fragmented environment model: II - Biological invasions and pulsating travelling fronts J. Math. Pures Appl. 848 11011146 .

[9] Berestycki H and Larrouturou B 1991 Quelques aspects mathématiques de la propagation des flammes prémélangées Nonlinear PDEs and their applications 220 65-129.

[10] Berestycki H and Nadin G 2012 Spreading speeds for one-dimensional monostable reaction-diffusion equations J. Math. Phys. 5311.

[11] Billingham J and Needham D J 1991 The development of traveling waves in quadratic and cubic autocatalysis with unequal diffusion rates I. permanent form of traveling waves Phil. Trans. $R$. Soc.. A 334 1-24.

[12] Bukhgeim A L and Klibanov M V 1981 Uniqueness in the large of a class of multidimensional inverse problems Soviet Mathematics-Doklady 24 244-247.

[13] Cantrell R S and Cosner C 2003 Spatial ecology via reaction-diffusion equations John Wiley \& Sons Ltd, Chichester, UK.

[14] Choi J 2003 Inverse problem for a parabolic equation with space-periodic boundary conditions by a Carleman estimate Inverse. Ill-posed Problems 11 (2) 111-135.

[15] Chouli M, E. M. Ouhabaz and M. Yamamoto 2006. Stable determination of semilinear term in a parabolic equation. Commun. Pure Appl. Anal.,5, 447-462.

[16] Cristofol M, Garnier J, Hamel F and Roques L 2011 Uniqueness from pointwise observations in a multi-parameter inverse problem Comm. Pur. Appl. Anal. 11 1-15. 
[17] Cristofol M and Roques L 2008 Biological invasions: deriving the regions at risk from partial measurements Math. Bio. sci. 215 158-166.

[18] Cristofol M and Roques L 2012 An inverse problem involving two coefficients in a nonlinear reaction-diffusion equation C. R. Acad. Sci. Paris. I 350 469-473.

[19] Cristofol M and Roques L 2013 Stable estimation of two coefficients in a nonlinear Fisher KPP equation Inverse Problems 29095007.

[20] DuChateau P and Rundell W 1985 Unicity in an inverse problem for an unknown reaction term in a reaction-diffusion equation J. Differ. Equs 59 155-164.

[21] Egger H, Engl H W and Klibanov M V 2005 Global uniqueness and Hölder stability for recovering a nonlinear source term in a parabolic equation Inverse Problems 21 271-290.

[22] Freidlin M and Gärtner J 1979 On the propagation of concentration waves in periodic and random media Sov. math - Dokl. 20 1282-1286.

[23] Fursikov A V and Imanuvilov O Y 1996 Controllability of Evolution Equations Lecture Notes Series 34 Seoul National University Korea.

[24] Gilbarg D and Trudinger N S 1983 Elliptic Partial Differential Equations of Second Order. Berlin:Springer).

[25] Hamel F 2008 Qualitative properties of monostable pulsating fronts : exponential decay and monotonicity J. Math. Pures Appl 89 355-399.

[26] Hamel F, Fayard J and Roques L 2010 Spreading speeds in slowly oscillating environments Bulletin of mathematical biology $\mathbf{7 2} 1166-1191$.

[27] Hamel F, Nadin G and Roques L 2011 viscosity solution method for the spreading speed formula in slowly varying media Indiana Univ. Math J. 60 1229-1247.

[28] Hamel F and Roques L 2010 Fast propagation for KPP equations with slowly decaying initial conditions J. Differ. Equs 249 1726-1745.

[29] Hamel F and Roques L 2011 Uniqueness and stability properties of monostable pulsating fronts Journal of the European Mathematical Society 13 345-390.

[30] Immanuvilov O Y and Yamamoto M 1998 Lipschitz stability in inverse parabolic problems by the Carleman estimate Inverse Problems 14 1229-1245.

[31] Kaddouri I and Teniou D E 2013 Inverse problem for a parabolic equation with periodic and nonsmooth coefficients C. R. Acad. Sci. Paris. I 351 191-196.

[32] Kato T 1984 Perturbation theory for linear operators. (NewYork: Springer).

[33] Klibanov M V 2004 Global uniqueness of a multidimensional inverse problem for a nonlinear parabolic equation Inverse Problems 22 1003-1032.

[34] Kolmogorov A N, Petrovsky I G, and Piskunov N S 1937 Étude de l'équation de la diffusion avec croissance de la quantité de matière et son application à un problème biologique Bull. Univ. Etat Moscou, Ser. Int. A 1 1-26.

[35] Lorenzi A 1982 An inverse problem for a semilinear parabolic equation. Ann. Mat. Pura Appl. 131 $145-166$.

[36] Murray J D 2002 Mathematical Biology Third Edition. Interdisciplinary Applied Mathematics 17, (New York: Springer).

[37] Pilant M S and Rundell W 1986 An inverse problem for a nonlinear parabolic equation. Commun. Partial Differ. Eqns 11 445-457.

[38] Roques L and Cristofol M 2010 On the determination of the nonlinearity from localized measurements in a reaction-diffusion equation Nonlinearity $\mathbf{2 3}$ 675-686.

[39] Roques L and Cristofol M 2012 The inverse problem of determining several coefficients in a nonlinear Lotka-Volterra system Inverse Problems 28075007.

[40] Roques L, Garnier J, Hamel F, and Klein E K 2012 Allee effect promotes diversity in traveling waves of colonization Proc. Natl. Acad. Sci. USA 23 8828-8833.

[41] Roques L, Hamel F, Fayard J, Fady B, and Klein E K 2010 Recolonisation by diffusion can generate increasing rates of spread Theor. Popul. Biol., 77 205-212.

[42] Shigesada N and Kawasaki K 1997 Biological Invasions: Theory and Practice Oxford Series in 
Ecology and Evolution Oxford: Oxford University Press).

[43] Tort T and Vancostenoble T 2012 Determination of the insolation function in the nonlinear Sellers climate model Ann. I. H. Poincaré 29 683-713.

[44] Turchin P 1998 Quantitative analysis of movement: measuring and modeling population redistribution in animals and plants Sinauer, Sunderland, MA.

[45] Weinberger H F 2002 On spreading speeds and traveling waves for growth and migration in periodic habitat J. Math. Biol. 45 511-548.

[46] Xin J 1992 Existence of planar flame fronts in convective-diffusive periodic media Arch. Ration. Mech. Anal. 121 205-233.

[47] Xin J 2000 Front propagation in heterogeneous media SIAM Rev. 42 161-230. 